\title{
A LOCALLY SIMPLY CONNECTED SPACE AND FUNDAMENTAL GROUPS OF ONE POINT UNIONS OF CONES
}

\author{
KATSUYA EDA \\ (Communicated by Frederick R. Cohen)
}

\begin{abstract}
Let $C X$ be the cone over a space $X$. Let a space $X$ be first countable at $x$, then the following are equivalent: (1) $X$ is locally simply connected at $x ;(2) \pi_{1}((X, x) \vee(X, x), x)$ is naturally isomorphic to the free product $\pi_{1}(X, x) * \pi_{1}(X, x)$; (3) $\pi_{1}((C X, x) \vee(C X, x), x)$ is trivial. There exists a simply connected, locally simply connected Tychonoff space $X$ with $x \in X$, such that $(X, x) \vee(X, x)$ is not simply connected.
\end{abstract}

Griffiths [2] proved

Theorem 1 (H. B. Griffiths). Let a space $X$ be locally simply connected at $x \in X$ and also first countable at $x$. Then, for an arbitrary space $Y$ with $y \in Y$, the fundamental group $\pi_{1}((X, x) \vee(Y, y), x)$ of the one point union $(X, x) \vee$ $(Y, y)$ is naturally isomorphic to the free product $\pi_{1}(X, x) * \pi_{1}(Y, y)$.

In addition, in the same paper he proved that local simple connectivity is essential in this theorem. On the other hand the author [1] of the present paper has shown that the first countability in the theorem is also essential. In $\S 1$ we shall prove

Theorem 2. Let spaces $X, Y$ be first countable at $x, y$, respectively. Then the following are equivalent:

(1) At least one of $X$ and $Y$ is locally simply connected at $x$ or $y$, respectively;

(2) $\pi_{1}((X, x) \vee(Y, y), x)$ is naturally isomorphic to the free product $\pi_{1}(X, x) * \pi_{1}(Y, y)$

(3) $\pi_{1}((C X, x) \vee(C Y, y), x)$ is trivial, where $C X, C Y$ are the cones over $X, Y$, respectively.

Corollary 3. Let a space $X$ be first countable at $x$. Then the following are equivalent:

(1) $X$ is locally simply connected at $x$;

(2) $\pi_{1}((X, x) \vee(X, x), x)$ is naturally isomorphic to the free product $\pi_{1}(X, x) * \pi_{1}(X, x)$

(3) $\pi_{1}((C X, x) \vee(C X, x), x)$ is trivial.

Received by the editors February 27, 1990 and, in revised form, November 26, 1990.

1991 Mathematics Subject Classification. Primary 55Q20, 55Q52.

Key words and phrases. Fundamental group, locally simple, first countable, one point union, cone. 
This confirms a conjecture in [2, p. 187]. In $\S 2$ we prove

Theorem 4. There exists a simply connected, locally simply connected Tychonoff space $X$ with $x \in X$ such that $(X, x) \vee(X, x)$ is not simply connected.

This strengthens [1, Theorem 2], which asserts the existence of such a space locally simply connected only at $x$.

Now we state some definitions. A loop $f$ in a space $X$ is a continuous map from a closed interval $[a, b]$ to $X$ such that $f(a)=f(b)$, where $a \leq b$. For a loop $f, f^{*}:[a, b] \rightarrow X$ is the loop defined by $f^{*}(x)=f(a+b-x)$. The point $f(a)=f(b)$ is called the basic point of $f$. By a loop in a pointed space $(X, x)$, we mean a loop in $X$ with basic point $x$. For a subset $U$ of $X$, we say that a loop $f$ is in $U$ if the image of $f$ is included in $U$. Two loops $f, g$ are briefly said to be homotopic in $X$ if $f$ and $g$ have the same domain $[a, b]$ and there exists a homotopy in $X$ relative to $\{a, b\}$ [5]. For loops $f:[a, b] \rightarrow X$ and $g:[c, d] \rightarrow X$ with $f(b)=g(c), \quad f g:[a, b+d-c] \rightarrow X$ is the loop defined by $f g(\alpha)=f(\alpha)$ for $a \leq \alpha \leq b$ and $f g(\alpha)=g(\alpha-b+c)$ for $b \leq \alpha \leq b+d-c$. For a loop $f:[0,1] \rightarrow X$ with basic point $x,[f]$ is the member of the fundamental group $\pi_{1}(X, x)$ corresponding to $f$. For a loop $f:[a, b] \rightarrow X$, let $g:[0,1] \rightarrow X$ be the loop such that $g(\alpha)=f(a+\alpha(b-a))$, which is denoted by $f \equiv g$. We frequently confuse $f$ and $g$. Therefore, for loops $f, g$ with basic point $x,[f],[g]$ are members of $\pi_{1}(X, x)$ and $[f][g]=[f g]$ and $[f]^{-1}=\left[f^{*}\right]$ hold. For a continuous map $\varphi: X \rightarrow Y$ with $\varphi(x)=y$, the induced homomorphism from $\pi_{1}(X, x)$ to $\pi_{1}(Y, y)$ is denoted by $\varphi_{*}$. A space $X$ is locally simply connected at $x$, if for any neighborhood $U$ of $x$ there exists a neighborhood $V(\subset U)$ of $x$ such that any loop $f$ in $V$ is homotopic in $U$ to a constant loop. We define $(X, x) \vee(Y, y)$ as the quotient space of the topological sum of $X$ and $Y$ with the identification of $x$ and $y$ [4] and call it a one-point union of $X$ and $Y$ with the common point $x=y$. The cone $C X$ over a space $X$ is the quotient space of the cylinder $X \times[0,1]$ obtained by regarding $X \times\{1\}$ as one point $p_{X}$. We identify $X$ with the subset $X \times\{0\}$ of $C X$. In addition, in case $A \subset X$, we regard $C A$ as a subspace of $C X$. All spaces in this paper are Hausdorff. Undefined notions are usual ones $[4,5]$.

\section{LOCAL SIMPLE CONNECTIVITY}

The first proposition of this section was proved by Griffiths [2, Theorem 3], but the proof is rather long and uses first countability. Here, we give a short proof, a modified idea of which will be used in the proof of Theorem 2.

Proposition 5. For arbitrary spaces $X$ and $Y$ with $x \in X$ and $y \in Y$, the free product $\pi_{1}(X, x) * \pi_{1}(Y, y)$ naturally becomes a subgroup of the fundamental group $\pi_{1}((X, x) \vee(Y, y), x)$.

Proof. Let $i_{X}: X \rightarrow(X, x) \vee(Y, y)$ and $i_{Y}: Y \rightarrow(X, x) \vee(Y, y)$ be the inclusion maps. Joining the induced homomorphisms $i_{X *}$ and $i_{Y *}$, we get a homomorphism $h: \pi_{1}(X, x) * \pi_{1}(Y, y) \rightarrow \pi_{1}((X, x) \vee(Y, y), x)$. What we need to show is the injectivity of $h$. If a member of $\pi_{1}(X, x) * \pi_{1}(Y, y)$ is not the identity, it has a reduced word of nonzero length. Let $f_{1}, \ldots, f_{n}$ be loops in $(X, x)$ or in $(Y, y)$ such that $\left[f_{k}\right] \neq e(1 \leq k \leq n)$ and $f_{k}$ is in $X$ if and only if $f_{k+1}$ is in $Y(1 \leq k \leq n-1)$. To show that $f=$ 
$f_{1} \cdots f_{n}$ is not homotopic to the constant $x$ by contradiction, we suppose the contrary. There exists a homotopy $H$ from $f$ to the constant, i.e., $H(0, t)=$ $H(s, 0)=H(s, 1)=x, H(1, t)=f(t)$ for $0 \leq s, t \leq 1$. We may assume $\operatorname{dom}\left(f_{k}\right)=[(k-1) / n, k / n]$. Let $H^{-1}((X, x) \vee(Y, y) \backslash\{\bar{x}\})=\bigcup_{\lambda \in \Lambda} O_{\lambda}$, where $\left\{O_{\lambda}: \lambda \in \Lambda\right\}$ is a pairwise disjoint family of nonempty open connected subsets of $[0,1] \times[0,1]$. Next set $V_{k}=\bigcup\left\{O_{\lambda}: O_{\lambda} \cap[(k-1) / n, k / n] \times\{1\} \neq \varnothing\right\}$. Then $V_{k} \cap V_{k+1}=\varnothing$ for $1 \leq k \leq n-1$. In addition, it never occurs that $V_{k_{1}} \cap V_{k_{3}} \neq \varnothing$ and $V_{k_{2}} \cap V_{k_{4}} \neq \varnothing$ for $k_{1}<k_{2}<k_{3}<k_{4}$, because $O_{\lambda}(\lambda \in \Lambda)$ are connected open sets. Therefore, whether or not there exist $i<j$ such that $V_{i} \cap V_{j} \neq \varnothing$, there must exist $k$ such that $1 \leq k \leq n$ and $V_{k} \cap V_{m}=\varnothing$ for any $m \neq k$. Now define $H_{k}$ by

$$
H_{k}(s, t)= \begin{cases}x & \text { if }(s, t) \notin V_{k} \\ H(s, t) & \text { otherwise }\end{cases}
$$

Then $H_{k}$ induces a homotopy from $f_{k}$ to the constant, which is a contradiction.

By the words "naturally isomorphic" in Theorem 1 and Theorem 2(2), we mean that $h$ in the above proof is an isomorphism. The next lemma is also by Griffiths [2, Lemma 3.1] with [3]. Since we need a proof for a modified use later (i.e., in the last remark of the present paper), we outline its proof.

Lemma 6 [2, Lemma 3.1]. Let $A$ be a subset of a space $X$ such that $x \in A$. Let $Y$ be the jointed space $C A \cup X$ with the identification of $A$ in each of $C A$ and $X$, and let $i: A \hookrightarrow X, j: X \hookrightarrow Y$ be the inclusion maps.

Then, the induced homomorphism $j_{*}: \pi_{1}(X, x) \rightarrow \pi_{1}(Y, x)$ is surjective and $\operatorname{Ker}\left(j_{*}\right)$ is the minimal normal subgroup $N$ containing $i_{*}\left(\pi_{1}(A, x)\right)$.

Proof. There exists a strong deformation retract from $Y \backslash\left\{p_{A}\right\}$ to $X$. Let $B$ be a path-connected component of $x$ in $A$. Any loop $f$ with basic point $x$ goes into $C(A \backslash B)$ through $p_{A}$ and hence using a contraction to $p_{A}$ uniformly we get a loop $g$ in $C B \cup X$ homotopic to $f$. Since $B$ is path connected, $g$ is homotopic to a loop in $Y \backslash\left\{p_{A}\right\}$. Using the deformation retract, we get a loop in $X$ that is homotopic to $g$, which implies that $j_{*}$ is surjective. Since $i_{*}\left(\pi_{1}(A, x)\right) \leq \operatorname{Ker}\left(j_{*}\right)$ clearly, we only need to show $\operatorname{Ker}\left(j_{*}\right) \leq N$.

Let $f$ be a loop in $X$ such that $j_{*}([f])=e$. Then there exists a homotopy $H$ from $f$ to the constant $x$, i.e., $H:[0,1] \times[0,1] \rightarrow X, H(0, t)=$ $H(1, t)=H(s, 0)=x$, and $H(s, 1)=f(s)$ for $s, t \in[0,1]$. Let

$$
H^{\prime}(s, t)= \begin{cases}p_{A} & \text { if } H(s, t) \in C(A \backslash B), \\ H(s, t) & \text { otherwise. }\end{cases}
$$

Then $H^{\prime}$ is also a homotopy from $f$ to the constant $x$. Hence we may assume $\operatorname{Im}(H) \subset C B \cup X$. Let $K=H^{-1}\{p\}$ and $H^{-1}(C A \backslash A)=O$. Cover $K$ by open polygons so that the closures of them are still in $O$. By compactness of $K$, we get a finite subcover. Tracing the most outside edges of polygons we get finitely many piecewise linear loops $J_{1}, \ldots, J_{n}$ in $O$ such that $J_{i} \cap J_{j}=$ $\varnothing, \quad U\left(J_{i}\right) \cap U\left(J_{j}\right)=\varnothing(i \neq j)$, and $K \subset \bigcup_{i=1}^{n} U\left(J_{i}\right)$, where $U\left(J_{i}\right)$ is the open polygon surrounded by $J_{i}$. Connect $J_{i}(1 \leq i \leq n)$ to the upper edge $[0,1] \times\{1\}$ by piecewise linear paths that are disjoint from other $J_{j}$ 's as in Figure 1 on the next page. 


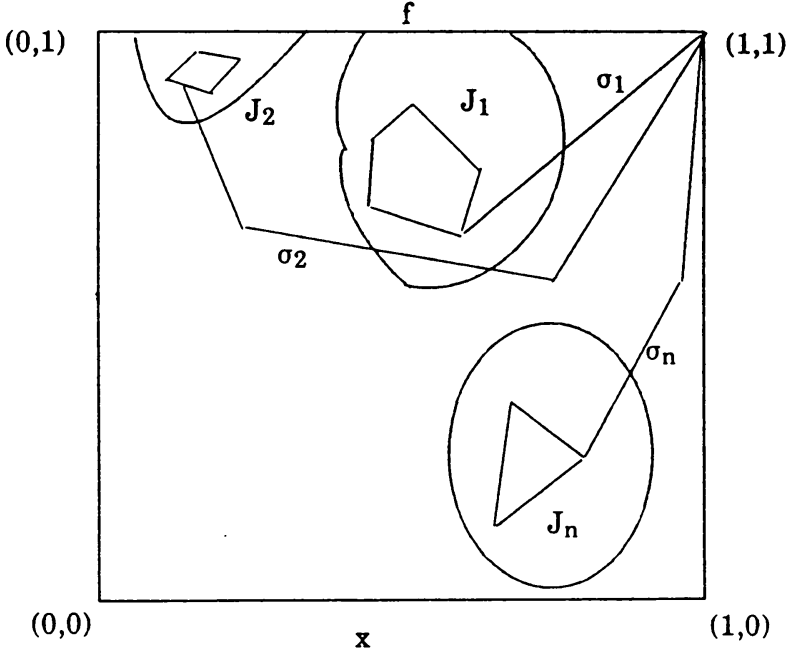

FIGURE 1

Then the path tracing $f, \sigma_{1}, J_{1}, \sigma_{1}^{*}, \ldots, \sigma_{n}, J_{n}, \sigma_{n}^{*}$ (counterclockwise on $\left.J_{i}\right)$ is a loop that is homotopic to the constant in $Y \backslash\{p\}$. Using path-connectivity of $B$ and the strong deformation retract from $Y \backslash\{p\}$ to $X$, we get loops $g_{1} \cdots g_{n+1}$ in $(X, x)$ and $a_{1} \cdots a_{n}$ in $(A, x)$ such that $f$ is homotopic $g_{1} a_{1} g_{2} \cdots g_{n} a_{n} g_{n+1}$ and $g_{1} g_{2} \cdots g_{n+1}$ to the constant $x$ both in $X$. Hence [ $f$ ] belongs to $N\left(i^{*}\left(\pi_{1}(A, x)\right)\right)$.

Proof of Theorem 2. The implication $(1) \rightarrow(2)$ is Theorem 1.

(2) $\rightarrow$ (3) This part is essentially [2, Lemma 3.3(ii)]. Use Lemma 6 twice and note that $\pi_{1}(X, x) * \pi_{1}(Y, y)$ naturally becomes a subgroup of the fundamental group $\pi_{1}((X, x) \vee(Y, y), x)$ by inclusion maps (Proposition 5). Then we get $\pi_{1}((C X, x) \vee(C Y, y), x) \simeq \pi_{1}((X, x) \vee(Y, y), x) / N$, where $N$ is the minimal normal subgroup containing $\pi_{1}(X, x) * \pi_{1}(Y, y)$. Hence, (2) implies (3).

(3) $\rightarrow$ (1) Let $U_{n}, V_{n}(n \in \mathbb{N})$ be basic open neighborhoods of $x, y$, respectively. Suppose that neither $X$ nor $Y$ is locally simply connected at $x$ or $y$, respectively. Then there exist an open neighborhood $U$ of $x$ and loops $f_{n}$ $(n \in \mathbb{N})$ with basic point $x$ such that $\operatorname{Im}\left(f_{n}\right) \subset U_{n}$ and $f_{n}$ is not homotopic in $U$ to the constant for each $n \in \mathbb{N}$. Similarly we get an open neighborhood $V$ of $y$ and loops $g_{n}(n \in \mathbb{N})$. Let $f$ be a loop in $(X, x) \vee(Y, y)$ such that $f(0)=f(1)=x, \quad f \mid\left[1 / 2^{m}, 3 / 2^{m+1}\right] \equiv f_{m}$, and $f \mid\left[3 / 2^{m+1}, 1 / 2^{m-1}\right] \equiv g_{m}$ for each $m$. The continuity of $f$ is assured by the constructions of $f_{n}, g_{n}$. Then there exists a homotopy $H$ in $(C X, x) \vee(C Y, y)$ from $f$ to the constant; i.e., $H:[0,1] \times[0,1] \rightarrow C X \cup C Y$ such that $H(0, t)=H(1, t)=H(s, 0)=x$ and $H(s, 1)=f(s)$ for $s, t \in[0,1]$. Let $O_{i}(i \in I)$ be open connected components such that $H^{-1}(C X \backslash\{x\})=\bigcup_{i \in I} O_{i}$ and $O_{i} \cap O_{j}=\varnothing$ for $i \neq j$. Likewise, let $H^{-1}(C Y \backslash\{x\})=\bigcup_{j \in J} P_{j}$. Set $I_{m}^{X}=\left(1 / 2^{m}, 3 / 2^{m+1}\right) \times\{1\}$ and $I_{m}^{Y}=\left(3 / 2^{m+1}, 1 / 2^{m-1}\right) \times\{1\}$. Let $V_{m}^{X}=\bigcup\left\{O_{i}: O_{i} \cap I_{m}^{X} \neq \varnothing\right\}$ and $V_{m}^{Y}=\bigcup\left\{P_{j}\right.$ : 
$\left.P_{j} \cap I_{m}^{Y} \neq \varnothing\right\}$. We say that $I_{m}^{X}$ is isolated if $V_{m}^{X} \cap V_{k}^{X}=\varnothing$ for $k \neq m$. Similarly for $I_{m}^{Y}$.

Case 1. There exist infinitely many $m$ such that $I_{m}^{X}$ is isolated. We get $i_{k} \in I(k \in \mathbb{N})$ such that $i_{k} \neq i_{k^{\prime}}$ for $k \neq k^{\prime}$ and $I_{i_{k}}^{X}$ is isolated. For $k \in \mathbb{N}$, let $H_{k}(\alpha)=H(\alpha)$ for $\alpha \in O_{i_{k}}$ and $H_{k}(\alpha)=x$ otherwise. Then $H_{k}$ induces a homotopy from $f_{i_{k}}$ the constant loop. There is a strong deformation retract from $C X \backslash C(X \backslash U)$ to $X$. Therefore there must exist $\alpha_{k} \in O_{i_{k}}$ such that $H\left(\alpha_{k}\right) \in C(X \backslash U)$. Since $V_{i_{k}}^{X}(k \in \mathbb{N})$ is pairwise disjoint, there exists a condensation point $\alpha^{*}$ of $\left\{\alpha_{k}: k \in \mathbb{N}\right\}$. Since we can get a subsequence of $\alpha_{k}(k \in \mathbb{N})$ that converges to $\alpha^{*}$, we assume $\lim _{k \rightarrow \infty} \alpha_{k}=\alpha^{*}$ for simplicity of notation. Then $H\left(\alpha^{*}\right)$ belongs to $C(X \backslash U)$, since it is closed. On the other hand, for each $k \in \mathbb{N}$ there exists a point $\beta_{k}$ on the segment from $\alpha_{k}$ to $\alpha^{*}$ such that $H\left(\beta_{k}\right)=x$. This implies $H\left(\alpha^{*}\right)=x$, which is a contradiction.

Case 2. Otherwise. Observe that if $V_{m}^{X} \cap V_{n}^{X} \neq \varnothing$ for $m<n$ then there exists $m \leq k<n$ such that $I_{k}^{X}$ is isolated or $I_{k}^{Y}$ is isolated. Hence the assumption implies the existence of infinitely many $m$ such that $I_{m}^{Y}$ is isolated. Now, the contradiction can be deduced in the same way as in Case 1.

Remark. According to [1, Theorem 2], the first countability is essential for the implication $(1) \rightarrow(2)$ in Theorem 2 and Corollary 2 . Here we show that the first countability is also essential for the inverse implication in them. Let $\mathbb{H}=$ $\bigcup_{n \in \mathbb{N}} C_{n}$ be the so-called Hawaiian earring, where $C_{n}=\left\{(x, y):(x-1 / n)^{2}+\right.$ $\left.y^{2}=1 / n^{2}\right\}$. We induce a finer topology using a nonprincipal ultrafilter $\mathscr{F}$ on $\mathbb{N}$. The topology at the points other than $(0,0)=O$ is the same as $\mathbb{H}$. For $X \in \mathscr{F}$ and open neighborhoods $U_{n}$ of $o$ in $C_{n}(n \notin X)$, let $O\left(U_{n}: n \notin\right.$ $X)=\bigcup_{n \in X} C_{n} \cup \bigcup_{n \notin X} U_{n}$. Induce a topology by these neighborhoods and let $\mathbb{H}_{\mathscr{F}}$ be the space. Though $\mathbb{H}_{\mathscr{F}}$ is not locally simply connected at $o, \mathbb{H}_{\mathscr{F}}$ shares the following property of the bouquet of infinitely many circles, which has the weak topology as a CW-complex. That is, any loop $f$ in $\mathbb{H}_{\mathscr{F}}$ is in a finite union of $C_{n}$ 's. Suppose not. There exists an infinite subset $X$ of $\mathbb{N}$ such that $\operatorname{Im}(f) \cap C_{n} \backslash\{o\} \neq \varnothing$ for each $n \in X$. Divide $X$ to two infinite subsets $X_{1}$ and $X_{2}$. Since $\mathscr{F}$ is an ultrafilter, at least one of them does not belong to $\mathscr{F}$, say $X_{1}$. Take $o \in U_{n} \subset C_{n}\left(n \in X_{1}\right)$ so that $\operatorname{Im}(f) \cap C_{n} \nsubseteq U_{n}$. Then there exists a condensation point $\alpha$ of the sequence of sets $f^{-1}\left(C_{n} \backslash U_{n}\right)\left(n \in X_{1}\right)$. Since $f(\alpha)=o$ but $O\left(U_{n}: n \notin \mathbb{N} \backslash X_{1}\right)$ does not intersect any $C_{n} \backslash U_{n}(n \in$ $\left.X_{1}\right)$, a contradiction occurs. Therefore, $\mathbb{H}_{\mathscr{F}}$ with $o$ satisfies the conclusion of [1, Proposition 3], which implies that $\pi_{1}\left(\left(\mathbb{H}_{\mathscr{F}}, o\right) \vee(Y, y), o\right)$ is naturally isomorphic to $\pi_{1}\left(\mathbb{H}_{\mathscr{F}}, o\right) * \pi_{1}(Y, y)$ for any space $Y$ with $y \in Y$.

The implication $(2) \rightarrow(3)$ in Theorem 2 and Corollary 2 holds without first countability as the proof shows. However, we do not know whether they are equivalent in general or not.

\section{CONSTRuction OF A LOCAlly SiMPly CONNECTED SPACE}

In [1] the author has shown the existence of a simply connected space $X$ with $x$ that is locally simply connected at $x$, but $(X, x) \vee(X, x)$ is not simply connected. Such a space cannot be first countable at $x$ by Griffiths's theorem (Theorem 1). The method of construction was to attach infinitely many copies 
of Griffiths's space, i.e., the cone on the Hawaiian earring, so that the resulting space would become locally simply connected at $x$. The method of construction for Theorem 3 is as follows. Regarding the construction in [1] as a procedure making a point locally simply connected, we will attach infinitely many copies of the space in [1] to points and iterate this process so that the resulting space becomes locally simply connected at all points.

For a set $X$, Seq $X$ is the set of all finite sequences of members of $X$, where a finite sequence of length $k$ is a function whose domain is the set $\{0,1, \ldots, k-1\}$. The length of a sequence $s$ is denoted by $\ln s$ and the empty sequence is denoted by \langle\rangle . For $k<\operatorname{lh} s, s\lceil k$ is the restriction of $s$ to the set $\{0, \ldots, k-1\}$. For a sequence $s$ with $\mathrm{lh} s=k$ and $x \in X$, $s *\langle x\rangle$ is the extension of $s$ by $x$, i.e., $\operatorname{lh} s *\langle x\rangle=k+1, s *\langle x\rangle(k)=x$, and $s *\langle x\rangle(i)=s(i)$ for $i<k$. The set of natural numbers is denoted by $\mathbb{N}$. Let $\Sigma$ be the set of triples of finite sequences $(\sigma, s, t)$ of the same length as the following: $\sigma \in \operatorname{Seq}(0,1), s, t \in \operatorname{Seq} \mathbb{N}$, and $s$ is nondecreasing. For $(\sigma, s, t),(\tau, p, q) \in \Sigma,(\tau, p, q)$ is an extension of $(\sigma, s, t)$ (denoted by $(\sigma, s, t) \preceq(\tau, p, q))$ if $\tau, p, q$ are extensions of $\sigma, s, t$, respectively. In addition, if $\operatorname{lh} \sigma<\operatorname{lh} \tau$, we say $(\tau, p, q)$ is a proper extension of $(\sigma, s, t)$ and denote it by $(\sigma, s, t) \prec(\tau, p, q)$.

For $m \in \mathbb{N}$, let

$$
X_{m}=\left\{(x, y, z): z=0,(x-1 / n)^{2}+y^{2}=1 / n^{2}, n \geq m\right\}
$$

be a so-called Hawaiian earring and

$$
Y_{m}=\left\{(x, y, z): 0<z \leq 1,(x-(1-z) / n)^{2}+y^{2}=(1-z)^{2} / n^{2}, n \geq m\right\}
$$

be the open cone on $X_{m}$. For an open subset $V$ of $X_{1}$ and open interval $(a, b)$, let

$$
\begin{array}{r}
V(a, b)=\left\{(x, y, z) \in Y_{1}: a<z<b,(x, y, z)\right. \text { is on the segment } \\
\text { between }(0,0,1) \text { and some point in } V\} .
\end{array}
$$

When $a<1<b$ and $V \neq \varnothing, \quad V(a, b) \cap Y_{m}$ is open in $Y_{m}$ iff $X_{m} \subset V$ for $m \in \mathbb{N}$. Now let $Y_{\langle\rangle,\langle\rangle}^{\langle\rangle}=Y_{1}$; for $(\sigma, s, t) \in \Sigma$, let $Y_{s, t}^{\sigma}$ be a copy of $Y_{m}$, where $m=s(\operatorname{lh} s-1)$; and denote the bijection by $i_{s, t}^{\sigma}: Y_{s, t}^{\sigma} \rightarrow Y_{m}\left(\subset Y_{1}\right)$ and $i_{s, t}^{\sigma}{ }^{-1}(x, y, z)$ by $(x, y, z)_{s, t}^{\sigma}$. Let $T=\bigcup\left\{Y_{s, t}^{\sigma}:(\sigma, s, t) \in \Sigma\right\}$ and $T_{s, t}^{\sigma}=\bigcup\left\{Y_{p, q}^{\tau}:(\sigma, s, t) \preceq(\tau, p, q) \in \Sigma\right\}$. We define a topology of $T$ as follows.

Case 1. $(x, y) \neq(0,0)$. The basic open neighborhoods of $(x, y, z)_{s, t}^{\sigma}$ are

$$
\begin{aligned}
U_{V, \varepsilon}\left((x, y, z)_{s, t}^{\sigma}\right)= & i_{s, t}^{\sigma}{ }^{-1} V(z-\varepsilon, z+\varepsilon) \\
& \bigcup \bigcup\left\{i_{p, q}^{\tau}{ }^{-1} V(0, \varepsilon):(\sigma, s, t) \prec(\tau, p, q),\right. \\
& |\tau(\operatorname{lh} \sigma)-z|<\varepsilon,|\tau(k)|<\varepsilon \text { for } \ln \sigma<k<\ln \tau\},
\end{aligned}
$$

where $V$ is an open subset of $X_{1}$ with $(x /(1-z), y /(1-z)) \in V$ and $(0,0) \notin$ $V$ and $0<\varepsilon<1-z$.

Case 2. $(x, y)=(0,0)$ and $z<1$. The basic open neighborhoods of 


$$
\begin{aligned}
& (0,0, z)_{s, t}^{\sigma} \text { are } \\
& U_{V, \varepsilon}^{\varphi, m}\left((0,0, z)_{s, t}^{\sigma}\right) \\
& =i_{s, t}^{\sigma}{ }^{-1} V(z-\varepsilon, z+\varepsilon) \\
& \cup \bigcup\left\{i_{p, q}^{\tau}{ }^{-1} V(0, \varepsilon):(\sigma, s, t) \prec(\tau, p, q),|\tau(\operatorname{lh} \sigma)-z|<\varepsilon ;\right. \\
& \text { If } p(k-1)<m \text { or } q(k-1)<\varphi(p(k-1)) \text {, } \\
& \text { then }|\tau(k)|<\varepsilon \text { for } \ln \sigma<k<\operatorname{lh} \tau \text {; } \\
& p(\operatorname{lh} \tau-1)<m \text { or } q(\operatorname{lh} \tau-1)<\varphi(p(\operatorname{lh} \tau-1))\} \\
& \cup \bigcup\left\{Y_{p, q}^{\tau}:(\sigma, s, t) \prec(\tau, p, q),|\tau(\operatorname{lh} \sigma)-z|<\varepsilon ;\right. \\
& \text { If } p(k-1)<m \text { or } q(k-1)<\varphi(p(k-1)) \text {, } \\
& \text { then }|\tau(k)|<\varepsilon \text { for } \operatorname{lh} \sigma<k<\operatorname{lh} \tau \text {; } \\
& p(\operatorname{lh} \tau-1) \geq m \text { and } q(\operatorname{lh} \tau-1) \geq \varphi(p(\operatorname{lh} \tau-1))\},
\end{aligned}
$$

where $V$ is an open subset of $X_{1}$ with $(0,0) \in V$ and $X_{m} \subset V, \quad 0<\varepsilon<$ $1-z, \varphi: \mathbb{N} \rightarrow \mathbb{N}$, and $m \in \mathbb{N}$.

Case 3. $(x, y)=(0,0)$ and $z=1$. The difference from Case 2 is the restriction of $V$ to $X_{1}$; that is, the basic open neighborhoods of $(0,0,1)_{s, t}^{\sigma}$ are

$$
\begin{aligned}
& \begin{array}{l}
U_{X_{1}, \varepsilon}^{\varphi, m}\left((0,0,1)_{s, t}^{\sigma}\right) \\
=i_{s, t}^{\sigma}{ }^{-1} X_{1}(1-\varepsilon, 1+\varepsilon)
\end{array} \\
& \cup \bigcup\left\{i_{p, q}^{\tau}{ }^{-1} X_{1}(0, \varepsilon):(\sigma, s, t) \prec(\tau, p, q),|\tau(\operatorname{lh} \sigma)-1|<\varepsilon ;\right. \\
& \text { If } p(k-1)<m \text { or } q(k-1)<\varphi(p(k-1)), \\
& \text { then }|\tau(k)|<\varepsilon \text { for } \operatorname{lh} \sigma<k<\operatorname{lh} \tau ; \\
& p(\operatorname{lh} \tau-1)<m \text { or } q(\operatorname{lh} \tau-1)<\varphi(p(\operatorname{lh} \tau-1))\} \\
& \begin{array}{r}
\cup\left\{Y_{p, q}^{\tau}:(\sigma, s, t) \prec(\tau, p, q),|\tau(\operatorname{lh} \sigma)-1|<\varepsilon ;\right. \\
\text { If } p(k-1)<m \text { or } q(k-1)<\varphi(p(k-1)), \\
\operatorname{then~}|\tau(k)|<\varepsilon \text { for } \ln \sigma<k<\operatorname{lh} \tau ; \\
p(\operatorname{lh} \tau-1) \geq m \text { and } q(\operatorname{lh} \tau-1) \geq \varphi(p(\operatorname{lh} \tau-1))\},
\end{array}
\end{aligned}
$$

where $0<\varepsilon, \varphi: \mathbb{N} \rightarrow \mathbb{N}$, and $m \in \mathbb{N}$.

When we do not make any specification, we assume that basic open neighborhoods $U_{V, \varepsilon}\left((x, y, z)_{s, t}^{\sigma}\right)$ and $U_{V, \varepsilon}^{\varphi, m}\left((0,0, z)_{s, t}^{\sigma}\right)$ satisfy the conditions as defined above. If basic open neighborhoods of $(x, y, z)_{s, t}^{\sigma}$ and $(u, v, w)_{p, q}^{\tau}$ intersect, then one of $(\sigma, s, t)$ and $(\tau, p, q)$ is an extension of the other. If $(0,0,1)_{p, q}^{\tau}$ belongs to $U_{V, \varepsilon}^{\varphi, m}\left((0,0, z)_{s, t}^{\sigma}\right)$ for $0<z<1$, then $X_{m} \subset V$ and $p(\operatorname{lh} \tau-1) \geq m$. Therefore, $i_{p^{\prime} \cdot q^{\prime}}^{\tau^{\prime}}-1 V(a, b)=i_{p^{\prime} \cdot q^{\prime}}^{\tau^{\prime}} X_{1}(a, b)$ for any extension $\left(\tau^{\prime}, p^{\prime}, q^{\prime}\right)$ of $(\tau, p, q)$. These assure that the above defines a topology of $T$. The following can be easily seen. $T_{s, t}^{\sigma}$ is open and homeomorphic to $T$. The bijection $i_{s, t}^{\sigma}$ is a homeomorphism. In the following we investigate the space $T$.

Lemma 7. $T$ is a Tychonoff space. 
Proof. It can be easily seen that $T$ is a Hausdorff space. It is easier to get the desired continuous map for a basic open neighborhood of $(x, y, z)_{s, t}^{\sigma}$ for $(x, y) \neq(0,0)$ or of $(0,0,1)_{s, t}^{\sigma}$ than the case for $(x, y)=(0,0)$ and $z<1$. Hence we only deal with $U_{V_{0}, \varepsilon_{0}}^{\varphi, m_{0}}\left(\left(0,0, z_{0}\right)_{s_{0}, t_{0}}^{\sigma_{0}}\right)$ for $z_{0}<1$. We may assume that $\varepsilon_{0}<\min \left\{z_{0}, 1-z_{0}\right\}$. Let $g: X_{1} \rightarrow\left[0, \varepsilon_{0}\right]$ be a continuous map such that $g(a, b)=0$ if $(a, b) \in X_{m_{0}}$ and $g(a, b)=\varepsilon_{0}$ if $(a, b) \notin V_{0}$. Define a map $h: T_{s_{0}, t_{0}}^{\sigma_{0}} \rightarrow[0,1]$ as follows, where we regard $x /(1-z)=y /(1-z)=0$ in case $z=1$ :

$$
\begin{gathered}
h\left((x, y, z)_{s, t}^{\sigma}\right) \\
=\left\{\begin{array}{r}
\max \left\{g(x /(1-z), y /(1-z)),\left|z-z_{0}\right|\right\} \\
\quad \text { if }(\sigma, s, t)=\left(\sigma_{0}, s_{0}, t_{0}\right) ; \\
\max \left\{g(x /(1-z), y /(1-z)), z,\left|\sigma\left(\operatorname{lh} \sigma_{0}\right)-z_{0}\right|,|\sigma(k)|:\right. \\
\left.\operatorname{lh} \sigma_{0}<k<\operatorname{lh} \sigma, s(k-1)<m_{0}, \text { or } t(k-1)<\varphi(s(k-1))\right\}, \\
\text { if }\left(\sigma_{0}, s_{0}, t_{0}\right) \prec(\sigma, s, t) \text { and } s(\operatorname{lh} \sigma-1)<m_{0} \\
\text { or } t(\operatorname{lh} \sigma-1)<\varphi(s(\operatorname{lh} \sigma-1)) ; \\
\max \left\{\left|\sigma\left(\operatorname{lh} \sigma_{0}\right)-z_{0}\right|,|\sigma(k)|: \operatorname{lh} \sigma_{0}<k<\operatorname{lh} \sigma,\right. \\
\left.s(k-1)<m_{0} \text { or } t(k-1)<\varphi(s(k-1))\right\}, \\
\text { if }\left(\sigma_{0}, s_{0}, t_{0}\right) \prec(\sigma, s, t), s(\operatorname{lh} \sigma-1) \geq m_{0}, \\
\text { and } t(\operatorname{lh} \sigma-1) \geq \varphi(s(\operatorname{lh} \sigma-1)) .
\end{array}\right.
\end{gathered}
$$

Let $f: T \rightarrow[0,1]$ be the map defined by

$$
f(\alpha)= \begin{cases}h(\alpha) & \text { if } \alpha \in T_{s_{0}, t_{0}}^{\sigma_{0}}, \\ \varepsilon_{0} & \text { otherwise. }\end{cases}
$$

Then, $f\left(\left(0,0, z_{0}\right)_{s_{0}, t_{0}}^{\sigma_{0}}\right)=0$ and $f(\alpha) \geq \varepsilon_{0}$ for $\alpha \notin U_{V_{0}, \varepsilon_{0}}^{\varphi, m_{0}}\left(\left(0,0, z_{0}\right)_{s_{0}, t_{0}}^{\sigma_{0}}\right)$. First we check the continuity of $h$. Let $\varepsilon$ be an arbitrary given positive real. In case $(x, y) \neq(0,0)$, take $V \ni(x /(1-z), y /(1-z))$ so that

$$
|g(a, b)-g(x /(1-z), y /(1-z))|<\varepsilon \text { for }(a, b) \in V \text {. }
$$

Then, $\left|h\left((u, v, w)_{p, q}^{\tau}\right)-h\left((x, y, z)_{s, t}^{\sigma}\right)\right|<\varepsilon$ for any $(u, v, w)_{p, q}^{\tau} \in$ $U_{V, \delta}\left((x, y, z)_{s, t}^{\sigma}\right)$. In case $(x, y)=(0,0)$ and $z<1$, take $V$ and $m \geq m_{0}$ so that $X_{m} \subset V$ and $|g(a, b)|<\varepsilon$ for $(a, b) \in V$. Then

$$
\begin{aligned}
&\left|h\left((u, v, w)_{p, q}^{\tau}\right)-h\left((0,0, z)_{s, t}^{\sigma}\right)\right|<\varepsilon \\
& \text { for any }(u, v, w)_{p, q}^{\tau} \in U_{V, \delta}^{\varphi, m}\left((0,0, z)_{s, t}^{\sigma}\right) .
\end{aligned}
$$

The continuity of $h$ at $(0,0,1)_{s, t}^{\sigma}$ is proved similarly. A remaining critical case to show the continuity of $f$ is that of $(0,0, z)_{s, t}^{\sigma}$ for $(\sigma, s, t) \prec$ $\left(\sigma_{0}, s_{0}, t_{0}\right)$. Take $m \geq m_{0}$ so that $s_{0}\left(\operatorname{lh} \sigma_{0}-1\right)<m$. Then $f\left((u, v, w)_{p, q}^{\tau}\right)=$ $\varepsilon_{0}=f\left((0,0, z)_{s, t}^{\sigma}\right)$ for $(u, v, w)_{p, q}^{\tau} \in U_{X_{1}, z_{0}-\varepsilon_{0}}^{\varphi, m}\left((0,0, z)_{s, t}^{\sigma}\right)$.

Lemma 8. Let $P$ be the set of all peaks of cones, i.e., $P=\left\{(0,0,1)_{s, t}^{\sigma}\right.$ : $(\sigma, s, t) \in \Sigma\}$. If the sequence $x_{n}(n \in \mathbb{N})$ of members of $P$ converges then there exists $p \in P$ such that $x_{n}=p$ for almost all $n \in \mathbb{N}$. 
Proof. Let $\lim _{n \rightarrow \infty} x_{n}=(x, y, z)_{s, t}^{\sigma}$ and $x_{n}=(0,0,1)_{s_{n}, t_{n}}^{\sigma_{n}}$. It is easily seen that $(x, y)=(0,0)$. Since $T_{s, t}^{\sigma}$ is open, almost all $\left(\sigma_{n}, s_{n}, t_{n}\right)$ are extensions of $(\sigma, s, t$,$) . Hence, we may only deal with the case where (\sigma, s, t) \prec$ $\left(\sigma_{n}, s_{n}, t_{n}\right)$ for $n \in \mathbb{N}$.

Case 1 . There exists $m$ such that $s_{n}(\mathrm{lh} \sigma)=m$ for infinitely many $n$. For an arbitrary $\varphi, U_{X_{1}, 1 / 2}^{\varphi, m+1}\left((0,0, z)_{s, t}^{\sigma}\right) \cap\left\{(0,0,1)_{p, q}^{\tau}: p(\mathrm{lh} \sigma)=m\right\}=\varnothing$, which contradicts $\lim _{n \rightarrow \infty} x_{n}=(x, y, z)_{s, t}^{\sigma}$.

Case 2. Otherwise. Taking a subsequence, we may assume that $s_{n}($ lh $\sigma)<$ $s_{n+1}(\operatorname{lh} \sigma)$. Take $\varphi$ so that $t_{n}(\operatorname{lh} \sigma)<\varphi(\operatorname{lh} \sigma)$. Then $U_{X_{1}, 1 / 2}^{\varphi, 1}\left((0,0, z)_{s, t}^{\sigma}\right) \cap$ $\left\{(0,0,1)_{p, q}^{\tau}: p(\operatorname{lh} \sigma)=m\right\}=\varnothing$, which contradicts $\lim _{n \rightarrow \infty} x_{n}=(x, y, z)_{s, t}^{\sigma}$.

Lemma 9. $T \backslash \bigcup\left\{T_{p, q}^{\tau}:(\sigma, s, t) \prec(\tau, p, q)\right\}(=X)$ is a strong deformation retract of $T \backslash\left\{(0,0,1)_{p, q}^{\tau}:(\sigma, s, t) \prec(\tau, p, q)\right\}(=Y)$ for $(\sigma, s, t) \in \Sigma$. Moreover, concerning basic open neighborhoods, $U_{V, \varepsilon}\left((x, y, z)_{s, t}^{\sigma}\right) \cap T_{s, t}^{\sigma}$ and $U_{V, \varepsilon}^{\varphi, m}\left((0,0, z)_{s, t}^{\sigma}\right) \cap T_{s, t}^{\sigma}$ are deformation retracts of $U_{V, \varepsilon}\left((x, y, z)_{s, t}^{\sigma}\right) \backslash P$ and $U_{V, \varepsilon}^{\varphi, m}\left((0,0, z)_{s, t}^{\sigma}\right) \backslash P$, respectively.

Proof. Let $H: Y \times[0,1] \rightarrow Y$ be the following map. For $x \in X, H(x, \alpha)=$ $x$. For $(\sigma, s, t) \prec(\tau, p, q) \in \Sigma$,

$$
\begin{aligned}
& H\left((x, y, z)_{p, q}^{\tau}, \alpha\right) \\
& =\left\{\begin{array}{l}
(x, y, z)_{p, q}^{\tau} \text { if } \sum_{i=1}^{\operatorname{lh} \tau-\operatorname{lh} \sigma} \frac{1}{2^{i}}<\alpha \leq 1, \\
\left(\frac{1-w}{1-z} x, \frac{1-w}{1-z} y, w\right)_{p \nmid k, q \uparrow k}^{\tau \uparrow k} \text { if } \sum_{i=1}^{k-1-\operatorname{lh} \sigma} \frac{1}{2^{i}}<\alpha \leq \sum_{i=1}^{k-\operatorname{lh} \sigma} \frac{1}{2^{i}}, \\
\left(\frac{1-\tau(\operatorname{lh} \sigma)}{1-z} x, \frac{1-\tau(\operatorname{lh} \sigma)}{1-z} y, \tau(\operatorname{lh} \sigma)\right)_{s, t}^{\sigma} \text { if } \alpha=0,
\end{array}\right.
\end{aligned}
$$

where

$$
w= \begin{cases}2^{k-\ln \sigma}\left(\alpha-\sum_{i=1}^{k-1} \frac{1}{2^{i}}\right) \tau(k) & \text { if } \ln \sigma \leq k<\operatorname{lh} \tau, \\ 2^{\operatorname{lh} \tau-\ln \sigma}\left(\alpha-\sum_{i=1}^{\ln \tau-1} \frac{1}{2^{i}}\right) z & \text { if } k=\ln \tau .\end{cases}
$$

It is a routine to check the continuity of $H$, hence we omit it. Since the restrictions of $H$ to the basic open neighborhoods in the second proposition are deformation retracts in themselves, the second proposition also holds.

Lemma 10. $T$ is locally simply connected.

Proof. Actually we prove that certain basic open neighborhoods are simply connected, and it is easily seen that they form neighborhood bases. By Lemma 9 we can see that $T$ is locally path connected. Therefore, we may choose basic points of loops freely to show that basic open neighborhoods are simply connected. The simple connectivity of $U_{V, \varepsilon}(x, y, z)_{s, t}^{\sigma}$ for a connected $V$ is easy in comparison with the following case. Hence, we only prove the simple connectivity of $U_{V, \varepsilon}^{\varphi, m}\left(\left(0,0, z_{0}\right)_{s, t}^{\sigma}\right) \quad(=U)$, where $V$ is connected and $\left\{(x, y):(x-1 / i)^{2}+y^{2}=1 / i^{2}\right\} \not \subset V$ for $i<m$. Let $f$ be a loop in $U$ and 
$f(0)=f(1)=\left(0,0, z_{0}\right)$. By Lemma 8 , there exist at most finitely many points in $P$ that belong to the image of $f$. Let $P \cap \operatorname{Im}(f)=\left\{(0,0,1)_{s_{k}, t_{k}}^{\sigma_{k}}: k=\right.$ $1, \ldots, n\}$. Next pick one of $\left(\sigma_{k}, s_{k}, t_{k}\right)(k=1, \cdots, n)$, say $\left(\sigma_{i}, s_{i}, t_{i}\right)$, so that the others are not proper extensions of $\left(\sigma_{i}, s_{i}, t_{i}\right)$. By Lemma 9, we get a loop $f_{1}$ in $U \backslash\left\{T_{p, q}^{\tau}:\left(\sigma_{i}, s_{i}, t_{i}\right) \prec(\tau, p, q)\right\}$ that is homotopic to $f$. Since $(0,0,1)_{s_{i}, t_{i}}^{\sigma_{\sigma_{i}}} \in U, T_{s_{i}, t_{i}}^{\sigma_{\sigma_{i}}} \subset U, s_{i}(d) \geq m$, and $t_{i}(d) \geq \varphi\left(s_{i}(d)\right)$, where $d=\ln \sigma_{k}-1$. Therefore, we get a loop $f_{2}$ so that $P \cap \operatorname{Im}\left(f_{2}\right)=$ $\left\{(0,0,1)_{s_{k}, t_{k}}^{\sigma_{k}}: k \neq i\right\}$ and $f_{2}$ is homotopic to $f_{1}$ in $U$. Iterating this process, we get a loop $g$ such that $P \cap \operatorname{Im}(g)=\varnothing, \operatorname{Im}(g) \subset T_{s, t}^{\sigma}$, and $g$ is homotopic to $f$ in the given neighborhood. Then $g$ is homotopic to a loop in $\left\{\left(x, y, z_{0}\right)_{s, t}^{\sigma}:\left(x /\left(1-z_{0}\right), y /\left(1-z_{0}\right) \in V\right\}\right.$. Since $V$ is connected and $\left\{(x, y):(x-1 / i)^{2}+y^{2}=1 / i^{2}\right\} \not \subset V$ for $i<m, g$ is homotopic to a loop in $\left\{\left(x, y, z_{0}\right)_{s, t}^{\sigma}:\left(x /\left(1-z_{0}\right), y /\left(1-z_{0}\right) \in X_{m}\right\}\right.$. Since $T_{s *\langle m\rangle, t *\langle\varphi(m)\rangle}^{\sigma *\left\langle z_{0}\right\rangle} \subset U$, we can conclude that $g$ is homotopic to the constant loop in $U$ and so is $f$.

Proof of Theorem 4. The space $T$ itself is like a basic open neighborhood, and hence the proof of Lemma 10 implies the simple connectivity of $T$. Let $t=(0,0, z)$ for $0<z<1$. Then $(T, t) \vee(T, t)$ is not simply connected. Since the proof is entirely the same as that of [1, Theorem 2], we omit it.

Remark. Here we remark that the fundamental groups of the spaces in Theorem 4 and [1, Theorem 2] and Griffiths's space [2] are isomorphic. Denote those spaces as $X_{1}, X_{2}, X_{3}$ with a distinguished point $t$, where $X_{1}=(T, t) \vee(T, t)$ and $t=(0,0, z)$ in the proof of Theorem 4. Let $T_{2}=\left\{(u, v, w) \in Y_{1}\right.$ : $w \geq z\} \cup \bigcup\left\{Y_{\langle m\rangle,\langle n\rangle}^{\langle z\rangle}: m, n \in \mathbb{N}\right\}$ and $T_{3}=\left\{(u, v, w) \in Y_{1}: w \geq z\right\}$. Then $X_{2}$ and $X_{3}$ are homeomorphic to the subspaces $\left(T_{2}, t\right) \vee\left(T_{2}, t\right)$ and $\left(T_{3}, t\right) \vee\left(T_{3}, t\right)$ of $X_{1}$, respectively. Let $\mathbb{H}=\left\{(u, v, w) \in Y_{1}: w=z\right\}$ and $i_{1}:(\mathbb{H}, t) \vee(\mathbb{H}, t) \hookrightarrow X_{1}, i_{2}:(\mathbb{H}, t) \vee(\mathbb{H}, t) \hookrightarrow X_{2}, i_{3}:(\mathbb{H}, t) \vee(\mathbb{H}, t) \hookrightarrow X_{3}$ be the inclusion maps.

Then, $\left(i_{3}\right)_{*}$ is surjective and $\operatorname{Ker}\left(\left(i_{3}\right)_{*}\right)$ is the minimal normal subgroup containing the free product $\pi_{1}(\mathbb{H}, t) * \pi_{1}(\mathbb{H}, t)$ by [2, Lemma 3.3], which can be proved using Lemma 6 twice. By Lemmas 8 and 9, we get a similar result to Lemma 6 concerning $i_{1}$ instead of $i_{3}$ and the same result for $\left(i_{1}\right)_{*}$. Since the corresponding properties of $T_{2}$ to Lemmas 8 and 9 also hold, we get the same result for $\left(i_{2}\right)_{*}$.

\section{ACKNOWLEDGMENT}

The author would like to say thanks to Topologists in Institute of Mathematics of University of Tsukuba, especially to T. Kaneto, T. Kimura, K. Kawamura, and K. Sakai. He also thanks the referee for his careful reading and suggestions.

\section{REFERENCES}

1. K. Eda, First countability and local simple connectivity of one point unions, Proc. Amer. Math. Soc. 109 (1990), 237-241.

2. H. B. Griffiths, The fundamental group of two spaces with a common point, Quart. J. Math. Oxford (2) 5 (1954), 175-190.

3. Math. Oxford (2) 6 (1955), 154-155. 
4. S. T. Hu, Homotopy theory, Academic Press, New York and London, 1959.

5. E. H. Spanier, Algebraic topology, McGraw-Hill, New York and San Francisco, 1966.

Institute of Mathematics, University of Tsukuba, Tsukuba 305, Japan

E-mail address: a906072@sakura.cc.tsukuba.ac.jp 